\title{
Análisis de evolución de la asistencia técnica y el fomento de cooperativas rurales en Colombia*
}

\author{
Assistance evolution analysis technique and the promotion \\ of cooperatives rural in Colombia
}

\author{
Análise da evolução da assistência técnica e promoção \\ de cooperativas rural na Colômbia
}

\section{Amanda Vargas-Prieto ${ }^{1}$ César Sánchez-Álvarez ${ }^{2}$}

Recibido: 22 de diciembre de 2018
Aceptado: 15 de diciembre de 2019
Publicado: 31 de enero de 2020

Cómo citar este artículo: Vargas-Prieto, A. y Sánchez-Álvarez, C. (2020). Análisis de evolución de la asistencia técnica y el fomento de cooperativas rurales en Colombia. Cooperativismo \& Desarrollo, 28(116)1-22. doi: https://doi.org/10.16925/2382-4220.2020.01.03

* Artículo de investigación. https://doi.org/10.16925/2382-4220.2020.01.03

1 Profesora de la Universidad del Magdalena

Doctorado en economía de la universidad de Burdeos, Francia, maestría en inteligencia económica y estrategias competitivas de la universidad de Angers, Francia, profesional en Administración de empresas del Politécnico Grancolombiano de Bogotá, Colombia.

Orcid: https://orcid.org/0000-0002-8508-6979

Correo electrónico: avargasp@unimagdalena.edu.co

2 Profesor investigador de la Universidad de La Salle.

Doctorado en Economía Social de la Universidad de Valencia.

Master en Cooperación al Desarrollo (esp. Planificación Integral del Desarrollo Local) de la Universidad de Valencia.

Licenciatura en Economía y Administración de Empresas Cooperativas y Organizaciones Sin Ánimo de Lucro de la Universidad de Bolonia - IT.

Licenciatura en Trabajo Social de la Universidad de Castilla la Mancha - ES.

Orcid: https://orcid.org/0000-0001-8274-6217

Correo electrónico: csa.puj@gmail.com 


\section{Resumen}

En la búsqueda de formalización de la economía y el ordenamiento territorial rural el legislador colombiano dio forma a las Zonas de Interés de Desarrollo Rural, Económico y Social (Zidres). Estas zonas son territorios de vocación agropecuaria que serán aprobados a partir de proyectos productivos individuales o asociativos. Según el artículo 1 de la Ley 1776 de 2016, la asistencia técnica será realizada por entidades encargadas desde un enfoque integral en el ciclo de los proyectos, garantizando la provisión de los paquetes tecnológicos, fomento de formas empresariales y servicios sociales básicos para el desarrollo rural. A través de una metodología cualitativa de análisis heurístico e histórico se identifican dos fases de un análisis en retrospectiva de un siglo de historia en la evolución de las instituciones que han promovido y fomentado la asistencia técnica en diferentes organizaciones y en el caso particular de las cooperativas agrarias. Los momentos identificados se corresponden a momentos históricos que han condicionado la formulación de las políticas públicas.

Palabras clave: Colombia, políticas públicas, asistencia técnica, cooperativas, economía social y solidaria. EconLit: P13, Empresas cooperativas; Q16, (R\&D - Agricultural Technology - Biofuel - Agricultural Extesion Services); Q18, (Agricultural Policy - Food Policy);

\section{Abstract}

In the search for formalization of the economy and the rural territorial planning the Colombian legislator shaped the Areas of Interest of Rural, Economic and Social Development (Zidres). These areas are territories agricultural vocation that will be approved from individual or associative productive projects.

According to article 1 of Law 1776 of 2016, technical assistance will be carried out by entities in charge from an integral approach in the project cycle, guaranteeing the provision of technology packages, promotion of business forms and basic social services for rural development. Through a methodology Qualitative heuristic and historical analysis identifies two phases of a retrospective analysis of a century of history in the evolution of the institutions that have promoted and promoted technical assistance in different organizations and in the particular case of agricultural cooperatives. The identified moments They correspond to historical moments that have conditioned the formulation of public policies.

Keywords: Colombia, public policies, technical assistance, cooperatives, social and solidarity economy. JEL code: P13, Cooperative Companies; Q16, (R\&D - Agricultural Technology - Biofuel - Agricultural Extesion Services); Q18, (Agricultural Policy - Food Policy)

\section{Resumo}

Na busca da formalização da economia e do planejamento territorial rural, o legislador colombiano moldou as Áreas de Interesse do Desenvolvimento Rural, Econômico e Social (Zidres). Essas áreas são territórios vocacionais agrícolas que serão aprovados a partir de projetos produtivos individuais ou associativos.

De acordo com o artigo $1^{\circ}$ da Lei 1776 de 2016, a assistência técnica será prestada pelas entidades responsáveis a partir de uma abordagem integral no ciclo do projeto, garantindo o fornecimento de pacotes de tecnologia, promoção de formas de negócios e serviços sociais básicos para o desenvolvimento rural. Através de uma metodologia, a análise heurística e histórica qualitativa identifica duas fases de uma análise retrospectiva de um século de história na evolução das instituições que promoveram e promoveram assistência técnica em diferentes organizações e no caso particular das cooperativas agrícolas. Os momentos identificados Correspondem a momentos históricos que condicionaram a formulação de políticas públicas.

Palavras-chave: Colômbia, políticas públicas, assistência técnica, cooperativas, economia social e solidária. Códigos JEL: P13, Empresas Cooperativas; Q16, (P\&D - Tecnologia Agrícola - Biocombustível - Serviços de Exportação Agrícola); Q18, (Política Agrícola - Política Alimentar) 


\section{Introducción}

El presente artículo se centra en la realidad rural colombiana que por más de cincuenta años ha estado sumergida en un conflicto armado con graves consecuencias. Un efecto del conflicto armado en Colombia ha sido el desplazamiento forzado producido por el despojo de tierras generando desplazamiento de la población rural a núcleos urbanos, siendo un fenómeno "masivo, sistemático, de larga duración y vinculado en gran medida al control de territorios estratégicos" (GMH, 2013, p. 71). El desplazamiento masivo ha aumentado la presión demográfica urbana generando una crisis humanitaria que afectó el 97 \% del territorio nacional (GMH, pp. 75-76), así como la destrucción de las relaciones sociales y el capital social de los territorios rurales.

El sector agropecuario colombiano siempre se ha caracterizado por la baja productividad y la débil competitividad. Esto se debe a diferentes carencias y problemáticas que arrastra históricamente Colombia, a saber, la deficiente infraestructura, el desigual acceso a la tierra y el conflicto armado, así como también la debilidad de las cadenas de valor (OCDE, 2015). Del informe de la OCDE se recoge que la innovación es fundamental para aumentar la competitividad de la economía agrícola, aunque presenta imperfecciones debido a "una escasa inversión privada, una baja oferta de conocimientos adaptados a la demanda, e importantes deficiencias en materia de capacidad y gobernanza" (OCDE, p. 7). Así bien, el informe de la OCDE plantea una serie de recomendaciones, en las reformas estructurales a largo plazo se recomienda llevar a cabo la revisión y evaluación de impacto de los programas e instrumentos de política para el sector agrícola que debe incluir los programas implementados por asociaciones privadas de productores con ayudas públicas (OCDE, p. 20).

Las diferentes negociaciones que han realizado los gobiernos colombianos con grupos insurgentes y paramilitares han visto la dejación de las armas y el inicio de procesos de participación democrática, es un ejemplo de este último la dejación de las armas del M-19 (Movimiento 19 de abril) el 8 de marzo de 1990, siendo liderados por Carlos Pizarro y dando vida al grupo político Alianza Democrática M-19, que quedaría tercero en las elecciones generales de 1990. El último proceso de diálogos y negociación ha sido concluido en octubre de 2016 entre el gobierno de Juan Manuel Santos y el grupo guerrillero FARC-EP, que vio la aprobación en la Cámara de Representantes del Acuerdo de Terminación del Conflicto después de la realización del plebiscito el 2 de octubre de 2016. La aprobación del Acuerdo de Terminación del Conflicto ha visto la realización y promulgación de normativa específica para asegurar el proceso de paz e iniciar una vía de posconflicto o posacuerdo gracias al mecanismo denominado Fast Track (vía rápida), para garantizar que las materias del acuerdo queden incluidas en la normativa legislativa. 
Del análisis realizado al macro del acuerdo para la terminación del conflicto y la construcción de una paz estable y duradera (2016), el punto 1 "Hacia un Nuevo Campo Colombiano Reforma Rural Integral", se reconoce que la efectividad y buen desarrollo de la Reforma Rural Integral depende de la participación de las comunidades, con espacios de participación para la transformación e incidencia en la "planeación, implementación y seguimiento de los diferentes planes y programas acordados" (Gobierno de Colombia y FARC-EP, 2016, p. 11). De manera específica a la Economía Solidaria y Cooperativa, el punto 1.3.3.1 establece "el fortalecimiento de las capacidades productivas y de las condiciones de acceso a los instrumentos de desarrollo rural (medios de producción, asistencia técnica, formación y capacitación, crédito y comercialización, entre otros)" (Gobierno de Colombia y FARC-EP, 2016, p. 28) orientando al desarrollo de la economía solidaria y cooperativa para estimular formas asociativas de trabajo basadas en la solidaridad y la cooperación.

Tradicionalmente la economía rural ha tenido aspectos culturales relacionados a las prácticas asociativas solidarias de los pobladores. Como se indica en el estudio de Misión Rural, la experiencia existente muestra que la asociatividad es la esencia misma de proyectos exitosos, así como la identificación de objetivos comunes y visiones compartidas de los territorios (Ocampo, 2014, p. 28). De igual manera, la asociatividad resulta exitosa porque se ha tenido un acompañamiento continuo de diferentes actores que han facilitado la transformación social en el contexto rural.

Según Orozco, Forero y Wills (2013) el campo colombiano se caracteriza por las prácticas de asociatividad para generar seguridad. Así bien, el informe para la "Misión para la Transformación del Campo" planteó la propuesta de políticas de Estado para que Colombia salde su deuda histórica con el campo como elemento esencial para construir la paz (Ocampo, 2014, p. 1). Los diálogos entre el gobierno de Juan Manuel Santos y las FARC-EP, realizados en la Habana desde el 26 de agosto de 2012 hasta el 24 de agosto de 2016, tuvieron desde el inicio la problemática del contexto rural y la sostenibilidad de la vida rural.

El primer informe presentado el 14 de octubre (2014) de "Misión para la Transformación del Campo" considera que en materia económica es necesario garantizar un desarrollo que permita el progreso de pequeñas, medianas y grandes empresas, incluyendo cooperativas y otras formas de asociaciones de productores (Ocampo, 2014, p. 2). Las pequeñas y medianas empresas en el campo colombiano son, en mayor medida, empresas familiares que subsisten en el medio rural gracias a prácticas eficaces de intercambio de factores productivos. La economía campesina tiene una difícil adaptación a nuevos parámetros de competitividad que han sido impulsados por los tratados de libre comercio (TLC). 
Los TLC han generado y están generando a nivel internacional una serie de cambios en los discursos políticos y su principal externalidad negativa -desempleopresente en países desarrollados está haciendo decantar un malestar hacia los procesos de globalización. En el caso de Colombia, el sector rural demostró un amplio malestar durante el Paro Agrario realizado el segundo semestre de 2013 y en concreto ha sido el primer punto de negociación en los Acuerdos de La Habana, que ha tenido su planteamiento en el primer punto del Acuerdo de Terminación del Conflicto en Colombia, donde se propuso la realización de una reforma agraria.

El acuerdo que fue renegociado con los representantes del voto negativo que emergió en el Plebiscito del 2 de octubre de 2016, fue ratificado en el Senado y en el Congreso a finales del mes de noviembre de 2016. Éste identifica diversos temas que no suponen la inmediata paz en Colombia, sino más bien implica definir nuevas políticas públicas orientadas a incentivar el desarrollo asociativo en el medio rural.

En concreto, la unión de las comunidades campesinas ha sido evidenciada desde un enfoque de asociatividad, que podría recogerse desde el enfoque de la economía social y solidaria. Pero las relaciones campesinas no han sido consideradas desde el análisis de las prácticas de solidaridad y la conformación de organizaciones formales o informales que se reconocen en la Ley 79 de 1988 de Cooperativas y la Ley 454 de 1998 de la economía solidaria.

El instrumento planteado como análisis de política pública especifica es la Ley 1776 de 2016, por la cual se crean y se desarrollan las ZIDRES. Esta ley plantea la creación de proyectos productivos en las zonas vulnerables -aislamiento de centros urbanos, altos costos de adaptación productiva, baja densidad poblacional, altos índices de pobreza y carencia de infraestructuras-. Estas zonas deberán promover e implementar los recursos necesarios para hacer competitiva el área rural a través de las actividades productivas rurales y políticas públicas "unificadas para favorecer la vida rural" (artículo 1, párrafo segundo). De igual forma, el artículo 3 identifica las tres formas jurídicas que pueden adelantar proyectos productivos en las ZIDRES, a saber, personas jurídicas, naturales o empresas asociativas. En el caso que se planteen proyectos asociativos se establecen requisitos específicos como puede ser la capacidad de asegurar "el suministro de servicios permanentes de capacitación empresarial y técnica, formación de capacidades y acompañamiento en aspectos personales y de dinámica grupal".

La misma ley hace hincapié en la participación asociativa del pequeño productor. En los artículos 16 y 17, se establece una garantía para la titulación de tierras para los pequeños productores que no cuenten con la titularidad de la propiedad (artículo 16). Así mismo, el artículo 17 hace referencia a la condición especial de proyectos 
productivos que vinculen población sin tierra, estableciendo la garantía de un proceso de tres años para hacerse titulares de la tierra, "de acuerdo al proyecto productivo y la capacidad financiera".

Así bien, la economía social y solidaria se podría presentar como instrumento para generar espacios de coordinación más eficientes, como se han identificado en los puntos del Acuerdo General (Gobierno de Colombia y FARC-EP, 2016). Es así que se resalta el tema del sector rural como una dimensión fundamental en las negociaciones del conflicto a nivel regional y local. Por tanto, resulta importante el análisis empírico y teórico de los emprendimientos sociales vinculados a la economía social y solidaria que emergen en Colombia, así como el estudio y análisis del ecosistema dirigido a fomentar y promocionar la asistencia técnica con una alta incidencia en la transformación del campo colombiano. Una de las organizaciones solidarias que tienen como objeto brindar el servicio de asistencia técnica a nivel mundial son las cooperativas agrarias. Sin embargo, a pesar de la legitimidad de las cooperativas al asumir la actividad de asistencia técnica, pocos estudios han examinado esta actividad (Faure et al., 2011).

Por los argumentos expuestos anteriormente y la necesidad de responder a la nueva reglamentación en un contexto de posacuerdo, el artículo se interesa responder a la siguiente pregunta, ¿cuáles son las variables históricas que han sido relevantes en la asistencia técnica dirigida al fomento y fortalecimiento de las cooperativas agrarias? Esta pregunta está orientada desde la necesidad de responder a los nuevos arreglos institucionales que demanda la nueva ley ZIDRES.

\section{Marco teórico}

El artículo recoge las bases del neoinstitucionalismo económico para analizar la estructura de las políticas públicas y los agentes individuales y grupales. El enfoque utilizado en este trabajo se espeja en los planteamientos de los trabajos llevados a cabo por Ronald Coase (1960), Douglass Cecil North (1990) y Oliver Williamson (1989).

Los trabajos de los anteriores autores demuestran cómo el funcionamiento del libre mercado depende de los arreglos institucionales, así como del rol de las instituciones en la disminución de los costos de transacción ya que disminuyen las asimetrías informativas, haciendo que los comportamientos de los agentes sean predecibles. Así bien, el artículo plantea un análisis para entender a los agentes y su capacidad de agencia y los límites de las políticas públicas. En otras palabras, el foco se encuentra en la tensión existente entre el principal - gobierno- y la estructuras 
- políticas públicas - que son creadas. Alcanzando así un análisis de cómo el libre mercado dependerá de: 1) los arreglos institucionales, y 2) el rol de las instituciones en la disminución de los costos de transacción para que los comportamientos de los agentes sean predecibles (Sánchez Álvarez, 2016).

Por tanto, el artículo se cuestiona por las reglas y normas que se están diseñando en Colombia durante un periodo definido como posacuerdo. De igual manera, se pone atención en los sistemas de gobierno que administraran las relaciones económicas, desde el análisis del cambio institucional y del desempeño económico (North, 1990), que han sido planteados en el Acuerdo Final de Terminación del Conflicto.

Se plantea la base para analizar los posibles costos de transacción (Coase, 1945; Williamson, 1989) que podría aumentar sí individuos con racionalidad limitada interactúan en estructuras complejas, con el riesgo de aumentar el oportunismo de los agentes. De otra forma, los agentes que se encuentran ante situaciones con altos costos de transacción podrían privilegiar el intercambio en condiciones fuera del mercado y por tanto, es necesario pensar en aquellas circunstancias determinadas que deberían estimular los controles de los intercambios, ayudando a monitorear los intercambios y asegurar que se cumpla el contrato entre las partes.

Desde el enfoque institucionalista se puede generar un marco teórico basado en la elección racional, donde el centro de la cuestión es la producción de equilibrios estables ante altos costos de transacción.

Siguiendo la obra de North consideramos que las instituciones son las reglas de juego y son restricciones creadas para organizar los agentes, diseñando estructuras de incentivos. Desde esta perspectiva cognitiva, se asume el planteamiento de North (1990) que indica la necesidad de realizar un análisis desde las perspectivas normativas, históricas y de elección racional, para entender las reglas de juego y las instituciones:

Institutions are the rules of the game in a society or, more formally, are the humanly devised constraints that shape human interaction. In consequence the structure incentives in human exchange, whether political, social, or economic. Institutional change shapes the way societies evolve through time and hence is the key to understandig historical change. (North, 1990 p. 3).

Por tanto, bajo las consideraciones de North, este trabajo indagará cómo las reglas básicas del juego determinan el funcionamiento de cualquier sistema económico. Muchas de estas reglas de funcionamiento se hacen específicas a través de los 
derechos de propiedad que determinan quién debe tener el derecho a usar o recibir recursos. Así bien, se consideran las reglas de incentivos como sustitutivos de valores y normas, de tal forma que los individuos podrían maximizar su utilidad individual cayendo en la tragedia de los comunes (Hardin, 1968).

\section{Metodología}

Expuesto el marco teórico, el trabajo se orienta metodológicamente a identificar e interpretar las instituciones que han dado respuesta a problemas de equilibrio perverso generando la organización entorno a la asistencia técnica para las empresas cooperativas en Colombia.

Por tanto, es necesario analizar e interpretar las políticas públicas que han propiciado equilibrios justos sin caer en la tragedia de los comunes. Para esto, se parte de la necesidad de entender el contexto de políticas públicas que tengan en cuenta el beneficio de la cooperación social para la producción de valor público como plantea Ostrom (2005) considerando el rol del legislador y del gobierno en la resolución de problemas de coordinación de actores como se plantea en el objeto de la Ley 1776 de 2016 - o de las ZIDRES-, a saber:

Créanse las zonas de interés de desarrollo rural económico y social (ZIDRES) como territorios con aptitud agrícola, pecuaria y forestal y piscícola identificados por la Unidad de Planificación Rural Agropecuaria [...] que se establecerán a partir de planes de desarrollo rural integral en un marco de economía formal y de ordenamiento territorial, soportados bajo parámetros de plena competitividad e inserción del recurso humano en un contexto de desarrollo humano sostenible, crecimiento económico regional, desarrollo social y sostenibilidad ambiental (art. 1 ZIDRES) .

Para ello se plantea una metodología cualitativa de análisis de las políticas públicas que se han dado en Colombia en consonancia con las tendencias de la asistencia técnica a nivel internacional. En concreto, el diseño y formulación de políticas públicas así como su incidencia en el desarrollo de la asistencia técnica en Colombia.

El artículo utiliza como insumo los trabajos doctorales de los autores Vargas Prieto (2013) y Sánchez Álvarez (2016) y se plantea una reflexión interpretativa de las etapas históricas que tienen como objeto la asistencia técnica y el fomento cooperativo en Colombia. 


\section{Análisis histórico de las políticas públicas de fomento cooperativo agropecuario en Colombia y el desarrollo de la asistencia técnica en el siglo XX}

Para realizar un análisis organizado y sistemático de la formulación de la política pública orientado a establecer las relaciones entre el Estado, la economía social y solidaria y la asistencia técnica en Colombia durante el siglo XX y XXI, se propone un análisis por fases.

La primera fase corresponde a los inicios de la asistencia técnica y políticas públicas de fomento fiscal que se dirigían a promocionar cooperativas rurales y urbanas con beneficios fiscales, específicamente para aumentar la colonización agrícola durante los años treinta. Esta fase será considerada brevemente por ser una fase de iniciación de la asistencia técnica y por políticas públicas de fomento de las cooperativas con carácter instrumental. La política pública se diseñaba en función de garantizar el control y la vigilancia de las cooperativas. De esta forma, los diferentes autores y textos que han sido analizados concuerdan con la dimensión local del cooperativismo fomentado "desde arriba" o top-down (Sánchez Álvarez, 2016). Como resultado de estas políticas públicas se establece una emergencia de las empresas cooperativas agropecuarias y de otros tipos, como fueron el fomento de cooperativas de consumo, crédito y venta de productos (CEPAL, 1989). Así bien, la institucionalidad pública se caracteriza por constantes cambios de dirección, y por una marcada instrumentalización de las cooperativas, reconocidas como movimiento social afín a los objetivos del gobierno.

Con respecto a la asistencia técnica no se tiene información de una organización institucional de la misma en Colombia. A nivel mundial, de acuerdo a Haug (1999), la asistencia técnica se caracteriza por un enfoque convencional hacia abajo (topdown) donde se realiza el acompañamiento de los agricultores y su formación a través de la aplicación de dos métodos educativos propuestos por el Banco Mundial y la FAO respectivamente: training and visit y farmer field school. Así, este periodo se puede caracterizar por una asistencia técnica incipiente y políticas públicas inexistentes.

Expuesto lo anterior, a continuación se plantean dos fases que recogen la mitad del siglo XX y que pueden ser sintetizadas por orientaciones diversas. 


\section{Asistencia técnica por oferta (1960-1985)}

La fase que se propone a continuación está caracterizada por: 1) la conformación de instituciones públicas de fomento cooperativo agrario, 2) el impulso de las reformas agrarias a nivel internacional, y 3) una asistencia técnica especifica dirigida desde los principios de la revolución verde.

A partir de los años cincuenta se da un incremento e importancia de la planeación nacional, y se crea la Oficina de Planificación de la Presidencia de la República durante el gobierno de Laureano Gómez. La necesidad de planeación emerge desde las necesidades y brechas que vive Colombia entre zonas rurales y urbanas. Las zonas rurales y los pobladores rurales - campesinos minifundistas y asalariados- eran dependientes de la agricultura y sufrían de ingresos insuficientes.

Como impulso para la planeación económica en Colombia es importante el Informe "Bases de un programa de fomento para Colombia" realizado por solicitud del gobierno colombiano al Banco Internacional de Reconstrucción y Fomento, donde se identificó la necesidad de fomentar las cooperativas agropecuarias considerando su aumento cuantitativo y las funciones para llenar vacíos en el sector agropecuario colombiano.

La reestructuración de la Superintendencia de Cooperativas realizada en 1944 y la creación del Servicio Nacional de Aprendizaje (SENA) en 1957, coinciden con un incremento del número de las cooperativas debido también a su promoción en diversos sectores, pasando de 4 cooperativas con 1807 miembros en 1936 a 425 con 234392 miembros en 1960. Otro hito importante en materia de fomento cognitivo sobre las empresas cooperativas será el Decreto 1646 de 1954, donde se plantea la obligación de la educación cooperativa, y se crea una línea de trabajo educativo en las escuelas primarias, secundarias y profesionales.

En esta fase, se observa igualmente una fuerte incidencia de la planeación estratégica orientada al desarrollo del país. Es el caso de la influencia del "Programa Alianza para el Progreso" de J. F. Kennedy entre 1961 y 1963. Este programa generaría la estructura nacional para las reformas en Colombia, sobre todo la reforma agraria, la cual tuvo incidencia en el fomento de cooperativas a través de la legitimación por parte del Estado cuando se creó la Central Cooperativa para la Reforma Agraria en 1968 (CECORA) para especializar el fomento cooperativo agrario que realizaba el Instituto Colombiano de la Reforma Agraria (INCORA), creado en 1961.

De esta forma se identifica la asistencia técnica centralizada dirigida a las cooperativas agrarias debido a la reforma agraria realizada por la Ley 135 de 1961, que creó el INCORA y el Instituto Colombiano de Agricultura (ICA). Desde esta ley se inician 
los primeros boatos de reforma agraria en Colombia, se reconoce la importancia de la asistencia técnica y se crean líneas de crédito para el fortalecimiento de los mercados.

Para generar una mayor operatividad de la asistencia técnica, en 1968 se dio vida a las Unidades Municipales de Asistencia Técnica (UMATA), con el fin de garantizar la asistencia técnica directa rural agropecuaria buscando ampliar la cobertura, calidad y pertinencia del servicio de asistencia técnica, así como el seguimiento, orientación y acompañamiento en la prestación del servicio por parte de las entidades del orden departamental y nacional, en condiciones que permitan la libre escogencia por los beneficiarios de dichos servicios. De esta manera se desarrolló una asistencia técnica local, pero dirigida desde el gobierno, dinámica que duraría hasta 1975.

La creación del INCORA tenía los objetivos de repartir las tierras y otros factores de producción, así como ofrecer servicios al campesinado. Se crean también las entidades privadas para la reforma agraria, como la Asociación Nacional de Usuarios Campesinos -(ANUC) y el CECORA, como ha sido indicado anteriormente.

Un gran cambio que surge en esta fase fue el diseño e implementación de la nueva institucionalidad creada en torno al Plan de Desarrollo Rural Integrado (DRI) en 1976. El DRI pondría una atención particular en las empresas cooperativas, considerándolas como instrumento para llevar a cabo la Reforma Agraria y para ello se basaría en su fomento desde el CECORA.

La necesidad de una planificación específica se basa en la problemática identificada a inicios de los años setenta, sobre la formulación de políticas que identifican a las cooperativas como agentes de la reforma agraria. Así, por tanto, la legitimación de los nuevos procesos y escenarios de políticas aumentarían el grado de eficiencia de políticas públicas agrarias, industriales, de ahorro y crédito, entre otras, siendo diseñadas por los planes nacionales. Aunque algunos planes nacionales de desarrollo no reconocieran las cooperativas en el desarrollo social, sí fueron reconocidas para mejorar la cadena agropecuaria. Es el caso del CONPES 39, que reconoció la experiencia del INCORA en el fomento de cooperativas, organizaciones de acción comunal y vivienda (DNP, 1968). También el Instituto de Mercadeo Agropecuario (IDEMA) recibió competencias en materia de desarrollo cooperativo dirigido a las fases de producción, mercadeo y consumo, a través de un específico "Programa de Fomento Cooperativo" formulado en el CONPES 360 (DNP, 1969). El CONPES, anteriormente citado, realizó una programación en diversas regiones dirigida a fomentar las cooperativas agropecuarias. En el caso de Cundinamarca se indicó el objetivo de mejorar socialmente al campesinado por medio de organizaciones cooperativas y de acción comunal. 
Las políticas públicas del gobierno de Alfonso López Michelsen se caracterizarían por orientarse a disminuir o cerrar las brechas identificadas. En materia de cooperativas, el gobierno reformuló y presentó con más detalle el Plan de Desarrollo de Política Agraria en el documento CONPES 1314 de 1975, definiendo las transferencias que el INCORA debía realizar al CECORA. Así, la política pública de asistencia técnica a las cooperativas agropecuarias se fortaleció en 1979 cuando se firmó un convenio de tres años entre el IICA y CECORA para realizar un acompañamiento técnico de fortalecimiento con el objetivo de aumentar la capacidad de CECORA en el asesoramiento, promocionar y apoyar a las organizaciones de pequeños productores en la comercialización de sus productos.

En el año 1977, la evaluación del DRI evidenció la centralización operativa y administrativa de las actividades del CECORA como la causa de la evaluación parcialmente insatisfactoria, registrado en el documento CONPES 1476 (DNP, 1978a). El resultado se repetiría en 1978, siendo los campesinos asociados más significativos -15 581 campesinos en 53 formas asociativas-, se llegó a la conclusión que la actividad era insuficiente ya que no se había ejecutado el presupuesto previsto. En la evaluación realizada en 1978 se identificó que solo el 30 \% había sido ejecutado y fue necesario reformular las actividades del CECORA (DNP, 1978b).

En el siguiente plan nacional de desarrollo (Plan de Integración Nacional), diseñado por el gobierno de Julio César Turbay, se reformuló la política para que los pequeños productores aumentaran su productividad, el aumento del ingreso real y el mejoramiento de su nivel de vida. Para ello el gobierno, a través del DRI y del CECORA, planteó la consolidación de las formas asociativas de producción, procesamiento y comercialización en zonas rurales. EI PND hizo hincapié en el DRI para realizar una acción conjunta de financiación de crédito por parte de Banco Interamericano de Desarrollo (BID), del Banco Internacional de Reconstrucción y Fomento (BIRF) y de la Agencia Canadiense de Desarrollo Internacional (ACDI). Esto hizo que el DRI se convirtiera en una política clave para la producción de alimentos de consumo masivo, siendo un instrumento válido para adelantar políticas sociales, orientadas a favorecer la población de más bajos ingresos en el sector rural. De esta manera, el CECORA y el Servicio Nacional de Aprendizaje (SENA) tuvieron un rol central en el DRI. La comercialización sería una función del CECORA, en cambio la línea de organización y capacitación sería realizada a través del SENA.

En síntesis, el plan diseñado por el gobierno de Julio César Turbay, con una lógica continuista, impulsó las formas asociativas para generar desarrollo industrial en la producción de alimentos, fomentando la integración vertical de la producción por parte del DRI. 
El modelo de asistencia técnica en Colombia, durante esta fase, se asemeja al estilo de la asistencia técnica mundial, con sus respectivas características desde la dimensión macroeconómica. En particular, se analiza el rol de los campesinos y las asociaciones de cooperativas en su relación con diferentes instituciones especializadas. Es decir, la política pública conlleva una especialización de las instituciones de asistencia técnica, generando innovación institucional.

A nivel mundial se inician procesos de transferencia de tecnología en el sector rural. Su objetivo es la difusión de información y técnicas (información sobre los nuevos insumos agrícolas, la capacitación sobre tecnología poco conocida). Durante esta fase, los agricultores fueron considerados como aplicadores de nuevas técnicas desarrolladas por la investigación agrícola (Ryan y Gross, 1943). En el caso de la asistencia técnica en Europa se reparten las tareas entre diferentes instituciones para crear innovaciones técnicas relacionadas con la asistencia técnica (Goulet et al., 2009). Sin embargo, esta fase ha sido criticada por investigadores como Simondon (1989) y Rabardel (1995), mostrando que la innovación técnica cuando se apropia y se utiliza, está sujeta a un proceso de adaptación y transformación. Entonces, los agricultores no deben ser considerados como simples aplicadores de nuevas técnicas, o como fue el caso de Colombia, no debieron ser considerados como meros receptores de políticas públicas, sino más bien generar procesos de co-creación de la política pública con la participación de los gremios. Fomentando así las relaciones constructivas de instituciones innovadoras con el Estado.

Darré (1986) también destaca el papel de los agricultores (individual y colectivo) en la producción de conocimiento. De hecho, la producción de conocimiento y la adaptación de nuevas técnicas ayudan a hacer que estas innovaciones sean más aceptables para un grupo de agricultores y garantizan su operatividad en la práctica (Goulet et al., 2009).

En Colombia, esta fase se cerraría con los primeros atisbos de un proceso de concertación de fomento cooperativo por parte del Estado. Dirigido a formalizar y fortalecer agremiaciones cooperativas desde los años cincuenta. El rol de las agremiaciones se vería con mayor intensidad en los trabajos del Plan Nacional de Desarrollo Cooperativo (1981-1982) también conocido como PLANDECOOP y que tendría su formulación institucional con el Conpes 1916 de 1982.

\section{Asistencia técnica por demanda (1985-2001)}

La fase que se propone a continuación está caracterizada por: 1) la importancia de la cuestión ecológica entre 1985 a 1995, 2) el creciente interés por el desarrollo 
sostenible (Brundtland, 1987), y 3) la aplicación de normativas que promovían el respeto por el medio ambiente.

Durante esta fase los agricultores se enfrentaron a múltiples problemas relacionados con temas ambientales, a saber, el aumento de los costos de tratamiento de residuos (Millet, 2003), la aparición de nuevos requisitos regulatorios (Green et al., 1994), nuevas preocupaciones de los consumidores, el aumento de los requisitos técnicos de los productos y los procesos, el aumento de la contaminación global, los riesgos económicos causados por el agotamiento de los recursos naturales, entre otros. (Millet, 2003). El medio ambiente y el desarrollo sostenible condicionaron fuertemente la organización de la asistencia técnica.

A partid de 1995, se pueden reconocer ciertos aspectos significativos, siendo los más representativos a nivel mundial, la separación del Estado y la aparición de nuevos actores en la asistencia técnica agrícola (Organizaciones No Gubernamentales (ONG), cooperativas agrícolas, organizaciones de productores, consultores privados, entre otros).

El desarrollo institucional en esta etapa ha sido relativamente bien estudiado. Los diferentes trabajos anteriormente mencionados demostraron que no es posible validar un único modelo de la asistencia técnica y que, a medida que pasa el tiempo, la organización de esta actividad depende del contexto agrícola de cada país e incluso de cada explotación (Anderson y Feder, 2004; Haug, 1999; Qamar, 2005; Rivera, 2000; Rivera y Alex, 2004; Swanson, 2006).

En el caso colombiano, esta fase puede identificarse como un periodo de crisis para la asistencia técnica, observándose una ruptura entre la investigación y el desarrollo tecnológico que generó problemas de acceso a la oferta tecnológica, desarticulación con las universidades, incoherencia con la demanda regional y vacíos en la planeación estratégica de los mercados y de la producción (Benítez, 2002).

En relación a las políticas públicas de fomento para las empresas cooperativas se deben mencionar dos fases propuestas por Sánchez Álvarez (2016). La primera fase evidencia el fracaso de la política económica centralizadora -fase definida como la Integración hacia el desarrollo, 1983-1991- y demuestra la ineficiencia institucional del DRI, sobre todo en las decisiones tomadas después de la evaluación del CECORA. La segunda fase denominada "La Evolución hacia la Globalización" (1991-2002), se caracterizó por los nuevos cambios en la institucionalidad, basados en los arreglos de poder definidos por la nueva Constitución Política de Colombia (1991) consolidando las relaciones entre el Estado y las cooperativas, así como la apertura económica que vivió el país desde 1992. 
La Constitución Política de 1991 otorga un mayor reconocimiento a organizaciones solidarias que compartían principios relacionados con la centralidad del ser humano en la economía - como contraposición a la economía capitalista de corte neoliberal-. Este reconocimiento favorecería una primera propuesta cooperativa urbana en sectores económicos relevantes. A saber, ahorro y crédito, salud y organización del mercado laboral como atestigua la política pública diseñada por el gobierno de Ernesto Samper (Plan Nacional de Desarrollo "El Salto Social") con el desarrollo de las cooperativas de trabajo asociado entendidas como emprendimientos asociativos colectivos, para mejorar el empleo. Considerando la economía solidaria una estrategia para introducir el trabajo asociado en los procesos productivos (Ley 188 de 1995, artículo 10).

En lo referente a las medidas de fomento cooperativo en el sector rural, aparecen programas de fomento cooperativo de crédito para pequeños productores, como es el caso del documento Conpes 2745 de 1994, que plantea la "Política de modernización rural y desarrollo empresarial campesino". De otra forma, la asistencia técnica estuvo designada a las entidades privadas de gremios industriales, a saber, CORPOICA, CENICAFE; CENICAÑA, CONIF CENIPALMA y CENIACUA (Conpes 2786 de 1995).

En conclusión, las cooperativas se convierten en instrumentos que debían favorecer el modelo latifundista y el desarrollo financiero, los cuales habían sido fortalecidos gracias a haber ganado espacios de relaciones a través de la estructura y funciones del DANCOOP, lo que generó una fuerte simbiosis entre política de gobierno y el fomento cooperativo, a través de programas de promoción y el fomento de precooperativas.

De otra forma, las alianzas entre entidades gubernamentales y organizaciones no gubernamentales, así como las organizaciones multilaterales (OIT y PNUD) se centrarían en la promoción de empresas asociativas dentro del Plan Nacional de Microempresa formulado por el Conpes 2541 de 1991, el cual dio importancia al rol de los organismos no gubernamentales dentro de programas específicos, como fue en la formación empresarial. Estos programas estaban caracterizados por asociaciones público-privadas dirigidas a atender conatos de grupos de economía solidaria. Este plan tendría su continuidad con el apoyo de microempresas, tanto en las áreas urbanas como rurales. En el caso de las áreas rurales se fomentó el acceso por parte de las mujeres, acceso fundamentalmente al crédito por parte de pequeñas explotaciones (Conpes 2745 de 1994) que eran facilitados por organizaciones no gubernamentales.

La fase propuesta se caracteriza por un segundo documento Conpes dirigido a la economía solidaria desde una solicitud del "sector solidario" y que se dirigía a modernizar y fomentar el desarrollo de la economía solidaria en su conjunto. En concreto, el Conpes 2823 de 1995 recoge la necesidad de: 1) asistencia técnica para la 
solidaridad -orientado a prestar servicios tecnológicos, administrativos y de formación de profesional-, 2) integración para el desarrollo económico local participativo, 3) capitalización de los pequeños productores, y 4) modernización rural y desarrollo rural campesino. Este último se orientaba desde la conceptualización del rol de la "empresa solidaria" en la formulación de proyectos productivos, teniendo atención a las cooperativas creadas durante la reforma agraria del DRI y fomentadas por el CECORA.

Lo más destacado de esta fase en materia de fomento cooperativo fue el fenómeno que se surgió a través de la empresa asociativa. A través de la participación de organizaciones no gubernamentales se crearon precooperativas y cooperativas de trabajo asociado en diferentes sectores, pero con una alta incidencia en el sector agropecuario, siendo un fenómeno que contuvo los números de organizaciones de la economía solidaria cuando afectó la crisis financiera en Colombia, la cual impacto en la quiebra de cooperativas financieras y de ahorro (Sánchez Álvarez, 2016).

\section{Análisis reciente del fomento de asociatividad cooperativa rural en Colombia y la asistencia técnica en el siglo XXI}

Recogiendo la fase propuesta por Vargas Prieto (2013) sobre la evolución de la asistencia técnica, en el siglo XXI se puede caracterizar por su capacidad de crear conocimiento. Labarthe (2006) considera la asistencia técnica agrícola como una relación de servicio que permite la producción de conocimiento, donde las interacciones entre los técnicos y agricultores juegan un papel importante. Así, la asistencia técnica agrícola se define como una relación de servicio entre los técnicos y agricultores, generando una coproducción de conocimiento. La constitución de este conocimiento se logra a través de la interacción entre los diferentes actores de esta actividad, técnicos y agricultores.

La asistencia técnica en Colombia en esta fase se caracteriza por garantizar subsidios específicos. Es el caso del programa "Agro ingreso seguro" que fue creado en 2007 bajo la Ley 1133. La actividad de asistencia fue subsidiada en el 80 \%. Sin embargo, esta iniciativa no resultó viable por la falta de acompañamiento de la institución sobre todo a los pequeños productores.

En relación a las políticas públicas de fomento cooperativo debemos hacer referencia a las dos fases propuestas por Sánchez Álvarez (2016) que analizan las implicaciones y repercusiones en la economía solidaria como un sistema socioeconómico que surge durante el proceso de globalización económica. Colombia 
se ve involucrada en los mercados internacionales desde la apertura económica de los años noventa teniendo implicaciones macroeconómicas (Ocampo, 2014).

De igual manera, la institucionalidad se caracteriza por la estructura del DANCOOP que sería transformada en el Departamento Nacional de Economía Solidaria - DANSOCIAL con funciones de promoción más allá de las empresas cooperativas, orientándose al fomento y promoción de todas las formas de la Economía Solidaria.

EI DANSOCIAL vería una ulterior transformación en el año 2011 convirtiéndose en Unidad Administrativa Especial de Organizaciones Solidarias - UAEOS- con la correspondiente pérdida institucional para la formulación y diseño de las estrategias de política pública, esta competencia pasó al Ministerio del Trabajo.

En este periodo aparece el segundo Plan del gobierno de Juan Manuel Santos (Todos por un Nuevo País, 2014-2018) y de acuerdo a Sánchez Álvarez (2016) tiene implicaciones para la asociatividad, siendo considerada como un elemento fundamental para alcanzar los objetivos de la planeación nacional. Lo que implica reconocer a la UAEOS como la entidad de fomento nacional de la asociatividad solidaria, que junto con el Ministerio del Trabajo y Ministerio de Agricultura deberán construir programas que fomenten las organizaciones rurales. Así, se observa cómo los últimos años en el diseño y formulación de políticas públicas de fomento de cooperativas y otras empresas u organizaciones de la economía solidaria ha estado determinada por los diálogos entre el equipo negociador del Gobierno del presidente Juan Manuel Santos y los delegados de las FARC-EP que fundamentalmente tuvieron al centro la problemática del contexto rural y la sostenibilidad de la vida rural.

Respecto al interés de este artículo, la asociatividad en las comunidades campesinas no ha sido evidenciada desde un enfoque de emprendimiento social, sino más bien se ha dado un enfoque desde la Economía Solidaria basándose en su forma jurídica de empresa cooperativa.

En concreto, desde trabajos anteriores se puede concluir que las relaciones campesinas han sido consideradas desde las prácticas de solidaridad y la conformación de organizaciones formales o informales que se reconocen en la Ley 79 de 1988, como ley general de cooperativas y la Ley 454 de 1998, siendo esta última una clara ley marco para la Economía Solidaria en Colombia.

Por tanto, la Economía Solidaria se presenta como instrumento para generar espacios de coordinación más eficientes, como se han identificado en diferentes puntos del Acuerdo General (Gobierno de Colombia y FARC-EP, 2016). En este caso, se plantea a las empresas cooperativas como instrumento de articulación y entrega de políticas públicas a la ciudadanía (Sánchez Álvarez, 2016) y como herramienta para la difusión y organización de la asistencia técnica (Vargas Prieto, 2013). 


\section{Conclusiones}

El objetivo del presente artículo ha sido identificar las variables históricas que han sido relevantes en la asistencia técnica dirigida al fomento y fortalecimiento de las cooperativas agrarias ante la situación actual que vive el campo colombiano.

El artículo ha realizado un análisis de los periodos históricos con una propuesta de fases de análisis (ver anexo). En el siglo XX se han identificado tres fases, el siglo XXI puede ser considerado por una única fase de políticas públicas colombianas de fomento cooperativo relacionadas a la asistencia técnica de procesos de asociatividad rural. Estos han sido elementos fundamentales para contextualizar la evolución de la asistencia técnica en Colombia y se ha demostrado que el fomento de las cooperativas rurales ha estado descoordinado respecto a la asistencia técnica. Es decir, el fomento cooperativo realizado por el Estado colombiano no ha estado orientado a las cooperativas agropecuarias. Las adaptaciones de la normativa para reconocer la realidad socioeconómica del cooperativismo del país han relegado a un último lugar el fomento de cooperativas agropecuarias. Pero a pesar de una atención escasa al desarrollo agropecuario desde la asociatividad cooperativa, el análisis cualitativo realizado para contextualizar y caracterizar la asistencia técnica en fases históricas nos permite plantear que la primera fase o la fase inicial aparece en los años treinta y consiste en una estrategia top-down basada en el fomento de las cooperativas agrarias a través de la creación de asistencia técnica y políticas públicas enfocadas en beneficios fiscales. La segunda fase corresponde al periodo entre 1950 y 1985 y se caracteriza por un tipo de asistencia técnica y políticas públicas por oferta del gobierno. La tercera fase corresponde al periodo entre 1985 y 2001, esta fase se caracteriza por un posicionamiento del modelo cooperativo en la organización del mercado que se reconoce por políticas públicas y asistencia técnica por demanda de las empresas cooperativas y gremios cooperativos.

Por tanto, a través de un marco teórico basado en el neo institucionalismo económico y una metodología cualitativa se analizaron las políticas públicas y su incidencia en la evolución de la asistencia técnica en Colombia. Las tres fases fundamentales en el desarrollo de las políticas públicas y de la asistencia técnica, nos han planteado los elementos para una agenda de investigación actual en torno a los elementos necesarios para definir la asistencia técnica como un elemento que debe recoger el fomento de las empresas cooperativas. Así bien, se pueden identificar las siguientes variables que se precisa analizar, a saber, 1) cómo se debe realizar la transferencia tecnológica a empresas cooperativas, buscando ser efectivos en la asimilación y utilización de las innovaciones, 2) cuál es la capacidad instalada de las cooperativas agropecuarias respecto a procesos de sostenibilidad ambiental, 
siendo las certificaciones de comercio justo (Sánchez Álvarez, 2018) instrumentos de referencia, y 3) cómo las políticas públicas deben tener en cuenta la generación de conocimiento y su distribución desde la capacidad de entender las relaciones entre las personas asociadas en cooperativas agropecuarias y los agentes de la asistencia técnica, en otras palabras, cabe responder a modelos de asistencia técnica propios para las empresas cooperativas, entendiendo éstas como empresas asociativas en contextos de altas asimetrías informativas.

En fin, el artículo nos plantea el reto sobre la necesidad de caracterizar y orientar las políticas públicas para una asistencia técnica pertinente y coherente con la identidad de las empresas cooperativas agropecuarias que se pueden instalar en las Zonas de Desarrollo Rural, Económico y Social - ZIDRES.

\section{Referencias}

Anderson, J. R., Feder, G. (2004). Agricultural Extension: Good Intentions and Hard realities. The World Bank Research Observer, 19(1),21.

Benítez, V.R. (2002). Investigación y competitividad agropecuaria. Economía Colombiana y Coyuntura Política, 292, 92-100.

Brundtland G.H. (1987). Our Common Future, Rapport de la commission mondiale sur l'environnement et le développement de l'Organisation des Nations-Unies, 318.

CEPAL. (1989). Cooperativismo Latinoamericano. Santiago de Chile, Chile: CEPAL.

Coase, R. (1945). Price and Output Policy of State Enterprise: A Comment. The Economic Journal, 55(217), 112-113.

Congreso de Colombia (2016). Ley 1776 por la cual se crean y se desarrollan las zonas de interés de desarrollo rural, económico y social. (ZIDRES).

Darré, J.P. (1986). Comment les façons de faire et de penser se transforment: l'étude des réseaux de dialogue. Agriscope, 7, 143-151.

Darré, J.P. (1994). Pairs et experts dans l'agriculture: dialogues et production de connaissances pour l'action. Ramonville Saint-Agne, Erès éditeur. 
Departamento Nacional de Planeación. (1968). Documento CONPES - 39. Programa Sectorial para la Agricultura. Bogotá: Departamento Nacional de Planeación

Departamento Nacional de Planeación. (1969). Documento CONPES - 360. Plan de Inversiones Públicas 1969-1972 (Anexo Sector Agropecuario). Bogotá: Departamento Nacional de Planeación.

Departamento Nacional de Planeación. (1978a). Documento CONPES - 1476. Evaluación de la ejecución del programa DRI. Bogotá: Departamento Nacional de Planeación

Departamento Nacional de Planeación. (1978b). Documento CONPES - 1512. Tercer Evaluación de la Ejecución del Programa DRI. Bogotá: Departamento Nacional de Planeación

Departamento Nacional de Planeación. (1994). Documento CONPES - 2745. Política de modernización ruraly desarrollo empresarial campesino. Bogotá: Departamento Nacional de Planeación

Departamento Nacional de Planeación. (1995). Documento CONPES - 2786. Política de competitividad agropecuaria, pesquera y forestal. Bogotá: Departamento Nacional de Planeación.

Faure, G., Desjeux, Y., Gasselin, P. (2011). Revue bibliographique sur les recherches menées dans le monde sur le conseil en agriculture. Cahier Agricole, 20, 327-342.

GMH. (2013). Basta Ya! Colombia: Memorias de guerra y dignidad. Bogotá: Imprenta Nacional.

Gobierno de Colombia y FARC-EP (2016). Acuerdo Final para la terminación del conflicto y la construcción de una paz estable y duradera. La Habana. Recuperado de https://www.mesadeconversaciones.com.co

Goulet, F. (2009). Des oubliés si présents. Dans B. Hervieu, B. Hubert. (Eds.), Colloque de Cerisy. Sciences en campagnes. La Tour d'Aigues: éditions de l'Aube.

Green, K., McMeekin, A., Irwin A. (1994). Technological trajectories and R\&D for environmental innovation in UK firms, Futures, 26(10), 1047-1059.

Hardin, G. (1968). The Tragedy of the Commons. Science, 1162, 1243-1248.

Haug, R. (1999). Some leading issues in international agricultural extension, a literature review. Journal of Agricultural Education and Extension, 4, 263-274. 
Labarthe, P. (2006). La privatisation du conseil agricole en question. Evolutions institutionnelles et performances des services de conseil dans trois pays européens (Allemagne, France, Pays-Bas). France: Université de Marne-La-Vallée, Champs-sur-Marne.

Millet, D. (2003). Intégration de l'environnement en conception: l'entreprise et le développement durable. Hermès Science Publications, 150.

North, D. C. (1990). Instituciones, cambio institucional y desempeño económico. México : Fondo de Cultura Económica.

Ocampo, J. A. (Coord.) (2014). El campo colombiano: un camino hacia el bienestary la paz. Informe detallado de la misión para la transformación del campo. Bogotá: Departamento Nacional de Planeación.

OCDE (2015). Revisión de la OCDE de las Politicas Agricolas: Colombia 2015. Evaluación y Recomendaciones de Politica. (Resumen Ejecutivo). Recuperado de https://www.oecd.org/ countries/colombia/OECD-Review-Agriculture-Colombia-2015-Spanish-Summary.pdf

Orozco, L. E., Forero, C., y Wills, E. (2013). Inseguridad rural y asociatividad. Una investigación sobre violencia y formas organizativas en zonas de conflicto. Bogotá: Universidad de los Andes.

Ostrom, E. (2005). Understanding institutional diversity. New Jersey, USA: Princeton University Press.

Qamar, M. K., (2005). Modernizing National Agricultural Extension Systems: A practical guide for Policy-Makers of Developing Countries. Rome, Italy: Nations ed., Food Policy.

Sánchez Álvarez, C. (2016). La Legitimación del Cooperativismo y de la Economía Social y Solidaria en las Políticas Públicas de Iberoamérica. El fomento cooperativo desde el gobierno de la República de Colombia y la influencia de Naciones Unidas y la Organización Internacional del Trabajo (Del 1913 a la actualidad). Tesis. Universidad de Valencia, España. Recuperado de http://roderic.uv.es/handle/10550/53666

Sánchez Álvarez, C. (2018). Comercio justo y economía social y solidaria: historia y evolución de sus instituciones de fomento. Revista Equidad y Desarrollo, 30, 149-172.

Vargas Prieto, A. (2013). Le rôle du conseil agricole dans la gouvernance cooperative. Tesis. Universidad de Burdeos, Francia. Recuperado de http://www.theses.fr/2013BOR40040

Williamson, O. (1989). Las instituciones económicas del capitalismo. México: Fondo de Cultura Económica. 


\section{Anexo 1. Evolución de las instituciones de fomento cooperativo y caracterización} de la asistencia técnica ${ }^{3}$
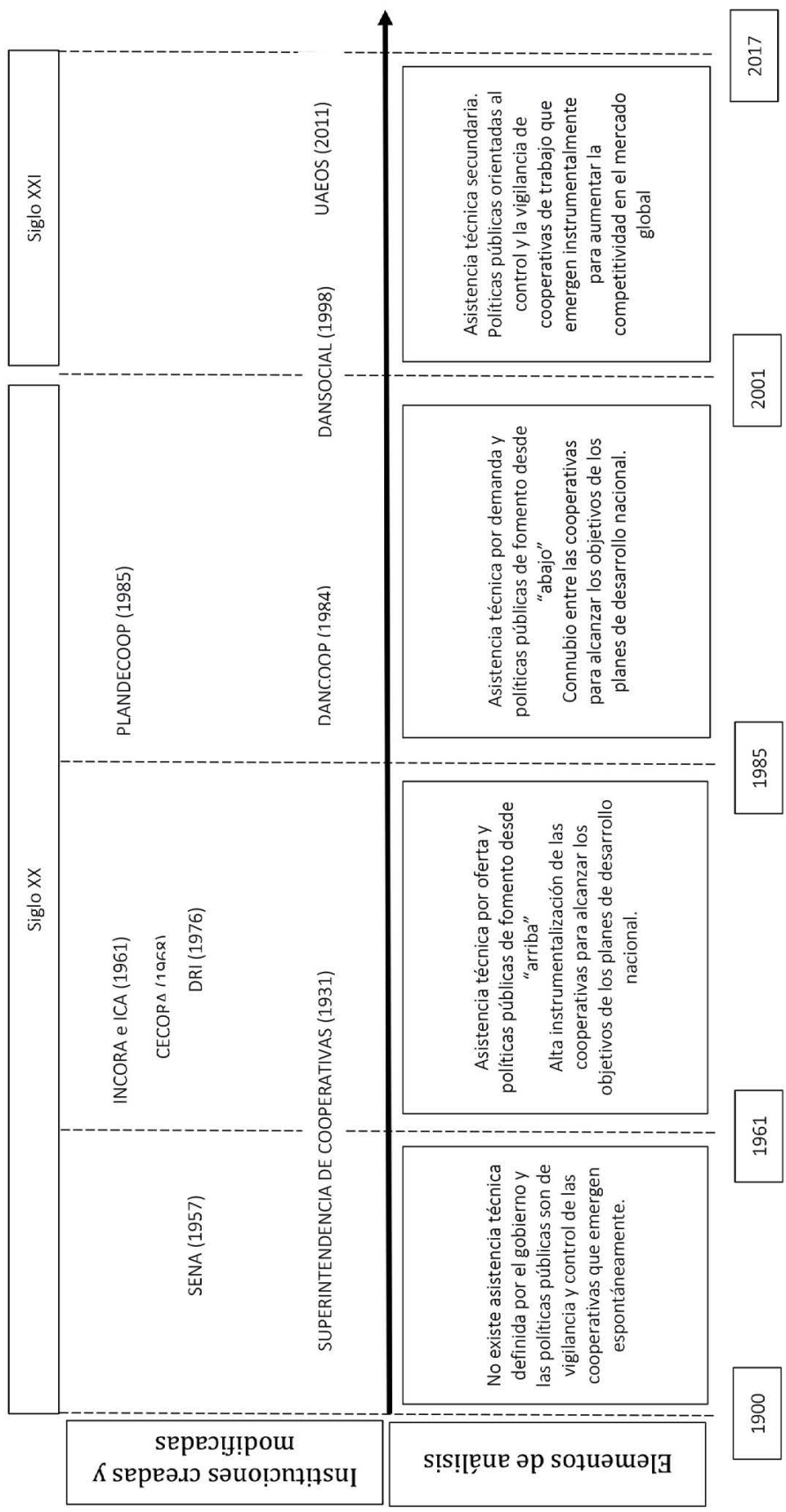

3 Entre paréntesis el año de creación de la institución de fomento cooperativo y acompañamiento en la asistencia técnica. 